\title{
Energy Efficiency Contours for Broadcast Channels Using Realistic Power Models
}

\author{
Amir Akbari, Member, IEEE, Fabien Héliot, Member, IEEE, Muhammad Ali Imran, Senior Member, IEEE, \\ and Rahim Tafazolli, Senior Member, IEEE
}

\begin{abstract}
Energy savings are becoming a global trend, hence the importance of energy efficiency (EE) as an alternative performance evaluation metric. This paper proposes an EE based resource allocation method for the broadcast channel $(\mathrm{BC})$, where a linear power model is used to characterize the power consumed at the base station (BS). Having formulated our EE based optimization problem and objective function, we utilize standard convex optimization techniques to show the concavity of the latter, and thus, the existence of a unique globally optimal energyefficient rate and power allocation. Our EE based resource allocation framework is also extended to incorporate fairness, and provide a minimum user satisfaction in terms of spectral efficiency (SE). We then derive the generic equation of the $\mathrm{EE}$ contours and use them to get insights about the EE-SE trade-off over the BC. The performances of the aforementioned resource allocation schemes are compared for different metrics against the number of users and cell radius. Results indicate that the highest EE improvement is achieved by using the unconstrained optimization scheme, which is obtained by significantly reducing the total transmit power. Moreover, the network EE is shown to increase with the number of users and decrease as the cell radius increases.
\end{abstract}

Index Terms-Broadcast channel, Contour, Convex optimization, Downlink, Energy efficiency.

\section{INTRODUCTION}

$\mathbf{E}$ NERGY consumption has recently become an integral aspect of wireless communications, both economically and environmentally. As a result of the increase in $\mathrm{CO}_{2}$ emissions from this sector, new stringent energy saving targets will have to be enforced for reducing these emissions. Alongside the environmental benefits, energy efficiency (EE) represents a huge economic opportunity for network providers who are also interested in reducing their electricity bills.

In order to reduce the power consumption of a mobile network, energy-efficient designs are being incorporated both in user equipments (UEs) and base stations (BSs). BSs consume the largest fraction of the energy in a mobile network [1], and accurately modelling their power consumption has recently attracted a lot of research interest, e.g. [2]. Modern BSs consume around $50 \%$ less power than traditional ones, which has been made possible by increasing the power efficiency of transceivers and replacing active air conditioners with freshair cooling systems in indoor sites [3].

The research leading to these results has been done within a joint project, partially supported by the European Communitys Seventh Framework Programme [FP7/2007-2013] under grant agreement no. 247733 - project EARTH, and by Huawei Tech. Co., Ltd, China.

The authors are with the Centre for Communication Systems Research, University of Surrey, Guildford, Surrey, GU2 7XH, UK (e-mail: a.akbari@surrey.ac.uk).
In the past, performance of wireless networks have been mainly characterized by the spectral efficiency (SE), e.g. [4]-[6], which is defined as the system throughput per unit bandwidth. In recent years, energy savings have become a global trend, hence the growing importance of the $\mathrm{EE}$ as a metric for network performance evaluation. The first study of EE for point to point links can be traced back to [7], which has been further extended in [8], where the capacity in bit-perjoule is given over a single link on flat fading and frequency selective additive white gaussian noise (AWGN) channels. In a single-cell context, the EE is studied in [9] and [10], [11] for flat fading and frequency selective channels respectively, where energy-efficient uplink communication is investigated for orthogonal frequency division multiple access (OFDMA) systems by either improving the utilization of the UE ([9], [10]) or BS ([11]) energy . These works however, assume orthogonal subcarriers and thus do not consider interference, simplifying the problem formulation.

Most existing system-level studies on EE have looked at EE optimization as a mean of measuring the efficiency of the communication link, with some also looking into the communication distance [12]. Such approaches are more suitable for mobile systems with a single objective, however, when multiple optimality measures are targeted, this approach is less effective. To tackle this, our previous work in [13] introduced EE contours as a novel way of obtaining the optimal operating point of the system based on multiple system requirements for the uplink channel. However, a user-centric EE formulation was given which did not address the EE of the single-cell system as a whole.

In this paper, we address the problem of optimizing the EE in the downlink of a single-cell system as in [10], [11], but in the presence of residual interference and with constraints on the total transmit power, fairness (through Jains fairness index [14]) and/or minimum user satisfaction (in terms of SE). The sophisticated linear power consumption model of [15] is considered in our analysis, which consists of two parts: an offset part, which is the power consumed in the system even when no data is transmitted, and a variable component which depends on the transmit power and amplifier efficiency.

Using standard convex optimization techniques, we show the concavity of the EE objective function, and existence of a unique globally optimal energy-efficient rate and power allocation. SE and EE are not consistent and in fact conflict with one another, such that a trade-off exists between these two quantities. The EE-SE trade-off in cellular networks acts as a guide for operators to obtain the best performance based 
on the primary target of the system, i.e. either towards an EE or SE target. Previous studies on the EE-SE trade-off are based on the sum-rate, which is why further investigation is required in order to obtain deeper insights into this tradeoff. In this regard, we derive the generic equation of the EE contours [13] for the broadcast channel (BC) and utilize them for analyzing and getting insights on the trade-off between EE and SE, with multiple constraints such as fairness and individual rate distribution of users.

The rest of the paper is organized as follows: Section II describes the system model and provides the SE formulation and total transmit power of the BS over the BC. Section III formulates the $\mathrm{EE}$ objective function as a function of SE, where standard convex optimization techniques are used to prove the concavity of the objective function. The EE objective function is initially tackled without any constraints, where an explicit closed-form of the optimal SE and EE are given. This is followed by the constrained optimization, where our EE based resource allocation scheme is compared against two other schemes; in addition to the total transmit power constraint, rate-fairness and per-user SE satisfaction are also considered. Section IV derives a closed-form function for the EE contours, which are used to identify optimal operating points based on different optimality measures. Section V provides our numerical results, which show that the highest EE improvements over the $\mathrm{BC}$ are obtained by using the porposed unconstrained optimization scheme. Finally, the paper is concluded in Section VI.

\section{SySTEM DESCRIPTION}

Let us consider the downlink of a single-cell, single-carrier, single-antenna, multi-user system, where a BS broadcasts a signal $x=\sum_{k=1}^{K} \sqrt{p_{k}} u_{k}$ to each of the $K$ users in the system, with $u_{k}$ and $p_{k}$ denoting the $k^{t h}$ unit normalized transmit symbol and power respectively, where $u_{k} \sim \mathcal{N}_{c}(0,1)$. The total transmit power at the $\mathrm{BS}, P$, can be expressed as:

$$
\mathrm{E}\left\{x^{\dagger} x\right\}=P=\sum_{j=1}^{K} p_{j}
$$

where $(.)^{\dagger}$ is the conjugate operator, and $E\{$.$\} stands for the$ expectation.

At each receiver, the signal from the $\mathrm{BS}$ is corrupted by an AWGN $n_{k}$ and is received as $y_{k}=h_{k} x+n_{k}$, where $h_{k}$ characterizese the $k^{t h}$ user channel. We assume that $n_{k}$ is a zero-mean complex Gaussian variable with variance $N=$ $N_{0} B$, where $N_{0}$ is the noise power spectral density and $B$ is the channel bandwidth.

The set of achievable rate points of the AWGN BC [16] can be computed using methods such as time-division (TD), frequency-division (FD), and superposition coding (SPC) [17]. The work in [18] showed that by ordering users based on their channel gains from strong to weak, the SPC rate region would in fact define the $\mathrm{BC}$ capacity region $\left(\mathcal{C}_{B C}\right)$. The SPC scheme operates best when users have more disparate channel quality, whereas in a symmetric case (when all users have equal channel gains), all spectrum sharing methods have the same rate region.
Assuming that SPC is employed at the transmitter, i.e. BS, and that the first user is encoded last (the user with the best channel, which is not affected by the interference of other users), the $k^{t h}$ user's SE can be expressed as

$$
S_{k}=\log _{2}\left(1+\frac{p_{k} g_{k}}{N+g_{k} \sum_{j=1}^{k-1} p_{j}}\right)
$$

and conversely,

$$
p_{k}=\left(2^{S_{k}}-1\right)\left(g_{k}^{-1} N+\sum_{j=1}^{k-1} p_{j}\right)
$$

where, $S_{k}=R_{k} / B, R_{k}$ is the $k^{t h}$ user's rate, and $g_{k}$ is the $k^{t h}$ user's channel gain, ordered such that $g_{1}>g_{2}>\cdots>$ $g_{K}>0$ and conversely, $g_{K}^{-1}>g_{K-1}^{-1}>\cdots>g_{1}^{-1}>0$.

Let us define $\alpha_{k}=g_{K+1-k}^{-1}-g_{K-k}^{-1}$ and $\alpha_{K}=g_{1}^{-1}$, which implies that $\alpha_{k} \geq 0, \forall k \in\{1, \ldots, K-1\}$ and $\alpha_{K}>0$. By inserting (3) into (1), $P$ can be re-expressed as:

$$
P=N\left[-g_{K}^{-1}+\sum_{i=1}^{K} \alpha_{i} \prod_{j=1}^{i} e^{X_{j}}\right]
$$

where, $X_{k}=S_{K+1-k} \ln (2), \forall k \in\{1, \ldots, K\}$.

\section{ENERGY-EFFICIENT DESIGN}

$\mathrm{EE}$ is defined as the number of bits transmitted per joule of energy [8], therefore, the overall $\mathrm{EE}$ of the $\mathrm{BC}$ is given as:

$$
E E_{T}=\frac{\sum_{k=1}^{K} R_{k}}{P_{T}}=\frac{B \sum_{k=1}^{K} S_{k}}{P_{T}}
$$

where, $P_{T}$ is the total consumed power, encompassing the BS transmit power as well as the circuit and processing powers of both BS and UE.

Recent studies on the energy consumption of cellular networks, including BSs, UEs, and the core network, revealed that around $80 \%$ of the energy required for the operation of a cellular network is consumed at BS sites [19]. In [15], a sophisticated power consumption model was defined, where a comprehensive power consumption analysis of the main power-hungry components of a BS were carried out and as a result, the relation between the relative radio frequency (RF) output power and BS power consumption was shown to be nearly-linear. Therefore, for studies which do not specifically focus on component-wise EE improvements, it is justified to consider a linear approximation for the power model [15], [20]. Using the aforementioned power consumption model, the total consumed power is formulated as:

$$
P_{T}=P_{c}+\beta P
$$

where, $\beta$ is the amplifier efficiency coefficient and $P_{c}$ denotes the total circuit and processing powers such that $P_{c}=P_{b s}+$ $\left(K P_{u e}\right)$, with $P_{b s}$ and $P_{u e}$ denoting the circuit and processing powers of the BS and UE respectively. 
Inserting (4) into (6), and the resulting equation into (5), we obtain that:

$$
E E_{T}=A \frac{\sum_{k=1}^{K} X_{k}}{\alpha_{0}+\sum_{k=1}^{K} \alpha_{k} \prod_{j=1}^{k} e^{X_{j}}}=A \frac{g(\mathbf{X})}{f(\mathbf{X})}
$$

where, $A=B(N \beta \ln (2))^{-1}, \alpha_{0}=\frac{P_{c}}{N \beta}-g_{K}^{-1}$ and $\mathbf{X}=$ $\left[X_{1}, \ldots, X_{K}\right]$.

The function $g(\mathbf{X})$ is the summation of a linear function and therefore concave, and the function $f(\mathbf{X})$ would be convex if $\alpha_{0} \geq 0$. However, it is not straightforward to conclude on the convexity or concavity of the ratio between a concave and convex function. Let $X_{k} \geq 0, \forall k \in\{1, \ldots, K-1\}$ and $X_{K}>0$. Therefore $g(\mathbf{X})>0$, and since $g(\mathbf{X})$ is concave and linear, $\ln (g(\mathbf{X}))$ is concave. Let $\alpha_{0} \geq 0$, then $f(\mathbf{X})>0$ and $E E_{T}>0$ such that:

$$
\ln \left(E E_{T}\right)=\ln (A)+\ln (g(\mathbf{X}))+h(\mathbf{X})
$$

where, $h(\mathbf{X})=-\ln (f(\mathbf{X}))$ is a concave function, hence, $\ln \left(E E_{T}\right)$ is a concave function, and the problem of maximizing $E E_{T}$ can be solved via standard convex optimisation tools such as the interior-point method [21]. The proof of concavity of $h(\mathbf{X})$ is given in Appendix A.

\section{A. Unconstrained Optimization}

Knowing that $\ln \left(E E_{T}\right)$ is concave implies that there exists a unique globally optimal $\mathbf{X}$ value that maximizes $E E_{T}$ over its entire domain. Using the EE formulation given in (5) and some mathematical computations, the optimal SE of the $k^{\text {th }}$ user can be obtained as:

$$
S_{k}^{\star}= \begin{cases}\frac{\mathcal{W}_{0}\left(e^{-1}\left(\frac{g_{1} P_{\mathrm{c}}}{N \beta}-1\right)\right)+1}{\ln (2)} & \text { if } k=1 \\ 0 & \text { if } k \in\{2, \ldots, K\}\end{cases}
$$

where, $\mathcal{W}_{0}$ denotes the real branch of the Lambert function and $S^{\star}$ is the optimal value of $S$. The proof of (9) is given in Appendix B.

Moreover, $p_{k}^{\star}$ can be simply obtained by inserting (9) into (3). Therefore, the optimal EE over the BC is given by

$$
E E_{o p t}^{\star}=A\left(S_{1}^{\star} \ln (2)-1\right)\left[\frac{P c}{N \beta}-g_{1}^{-1}\right]^{-1} .
$$

\section{B. Constrained Optimization}

In the previous section, we have derived explicit expressions of the optimal users rate and power that maximizes the EE without any constraints. Here, we extend our EE based resource optimization framework based on multiple constraints as discussed in subsequent sections:

1) EE Maximization with Total Power Constraint: This criterion corresponds to scenarios where the objective is to maximize $E E_{T}$ by ensuring that the total transmit power is less than or equal to $P_{\max }$, and that the results are within the set of achievable SEs that are defined as a function of the channel gain and $P_{\max }$. The resulting optimization problem is defined as:

$$
\begin{array}{rl}
\max _{S} & E E_{T} \\
\text { s.t. } & 0 \leq \sum_{k=1}^{K} p_{k} \leq P_{\max } \\
& \left(S_{1}, S_{2}, \ldots, S_{K}\right) \in \mathcal{C}_{B C}\left(P_{\max }, \mathbf{g}\right)
\end{array}
$$

where, $\mathbf{g}=\left[g_{1}, g_{2}, \ldots, g_{K}\right]$ and $P_{\max }$ is the maximum transmit power of the BS, and constraint (13) specifies that all rate pairs should be chosen within the $\mathrm{BC}$ capacity region. We denote this resource allocation method as $\mathrm{EE}_{\max }$.

With respect to constraint (12), if $\sum_{k=1}^{K} p_{k}<P_{\max }$, then the problem is equivalent to the unconstrained case defined in the previous subsection. On the other hand, if $\sum_{k=1}^{K} p_{k}=$ $P_{\max }$, then the problem reverts to the SE optimization problem which has been thoroughly investigated in literature, e.g. [4], with the optimimum SE given as:

$$
S_{k}^{\star}= \begin{cases}\log _{2}\left(1+g_{1} P_{\max } / N\right) & \text { if } k=1 \\ 0 & \text { if } k \in\{2, \ldots, K\} .\end{cases}
$$

2) EE Maximization with Total Power Constraint and RateFairness: In cases where as well as maximizing the EE, SEfairness is also required, a fairness constraint is added to the optimization problem defined in the previous subsection, so that every user receives a fair share of the available resources. This scheme is denoted as $\mathrm{EE}_{\text {fair }}$, and is defined as:

$$
\begin{array}{rl}
\max _{S} & E E_{T} \\
\text { s.t. } 0 \leq \sum_{k=1}^{K} p_{k} \leq P_{\max } & \\
& \left(S_{1}, S_{2}, \ldots, S_{K}\right) \in \mathcal{C}_{B C}\left(P_{\max }, \mathbf{g}\right) \\
& \mathcal{J}\left(S_{1}, S_{2}, \ldots, S_{K}\right)=1
\end{array}
$$

where, $\mathcal{J}$ represents the Jain's fairness index which ranges from $\frac{1}{K}$ (the least fair) to 1 (most fair), and is defined as [14]:

$$
\mathcal{J}\left(S_{1}, S_{2}, \ldots, S_{K}\right)=\frac{\left(\sum_{k=1}^{K} S_{k}\right)^{2}}{K \sum_{k=1}^{K} S_{k}^{2}} .
$$

3) EE Maximization with Total Power and Minimum SE Constraints: Rate-fairness poses a hard constraint on the optimization process which can conflict with the main objective. Therefore it is reasonable to say that rate-fairness focuses on the per-user satisfaction rather than optimizing the overall EE of the system. In order to obtain a more practical usage model, a SE constraint per user is added to the optimization problem, such that a minimum user satisfaction is provided. This scheme is denoted as $\mathrm{EE}_{\mathrm{min}-\mathrm{SE}}$, and is formulated as:

$$
\begin{array}{rl}
\max _{S} & E E_{T} \\
\text { s.t. } & 0 \leq \sum_{k=1}^{K} p_{k} \leq P_{\max } \\
& \left(S_{1}, S_{2}, \ldots, S_{K}\right) \in \mathcal{C}_{B C}\left(P_{\max }, \mathbf{g}\right) \\
& S_{k} \geq S_{\min }, \forall k \in\{1, \ldots, K\}
\end{array}
$$


where, $S_{\min }$ is the minimum SE constraint per user.

\section{Optimality Measures Using EE Contours}

For a function of two variables along the $x, y$-plane, a contour line defines the curve along which the function $f(x, y)$ has a constant value [22]. The same idea can be implemented for the EE [13] of communication systems, where by using (5), the overall $\mathrm{EE}$ of the $\mathrm{BC}$ can be obtained for each value of $\mathbf{X}$. The collection of all SE points, $\mathbf{X}$, that lead to the same $\mathrm{EE}$ value defines an $\mathrm{EE}$ contour.

For a given EE target, denoted as $E_{C}$, the corresponding EE contour and SEs can be obtained using the following parametric function:

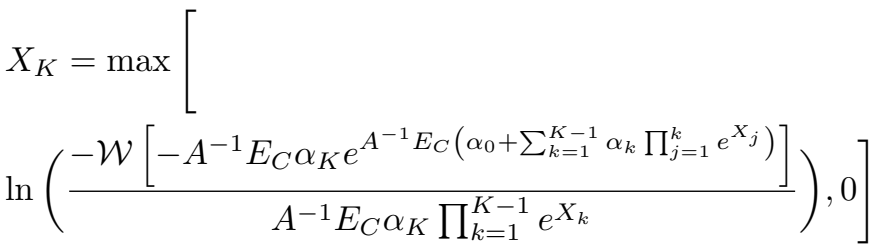

where, $\mathcal{W}($.$) is the Lambert function, \mathcal{W}=\mathcal{W}_{0}$ (real branch) if $-A^{-1} E_{C} \alpha_{K} \prod_{k=1}^{K-1} e^{X_{k}} \geq-1$ or $\mathcal{W}=\mathcal{W}_{-1}$ (negative branch) else. The proof of (24) is given in Appendix C.

Figure 1 depicts a 2-user example of the EE contours, which has been obtained by using (24), where a single snapshot is considered such that user 1 and 2 are 50 and 100 meters away from the BS, respectively. The $\mathrm{x}$-axis and $y$-axis represent the SE of user 1 and 2 respectively, where a linear relation is considered between the transmit power and total power consumption of the base station [15], with the rest of the simulation parameters summarized in Table I. The optimization problems defined in Section III are also demonstrated in Figure 1, where optimal operating points are highlighted.

The results clearly indicate that the maximum $\mathrm{EE}$ is achieved by only transmitting to the user closest to the BS, i.e. the user with the best channel gain, shown as point $\mathbf{A}$. This confirms the result that has been obtained in Section III, where the coordinates of point $\mathbf{A}$ can be computed by using (9). In order to incorporate fairness into the system, a line is drawn through the origin with a slope of +1 (line of fairness), where every point along this line will have equal SEs for both users, and the optimal $\mathrm{EE}$ when $\mathrm{SE}_{1}=\mathrm{SE}_{2}$ is depicted at point $\mathbf{B}$. Moving from point $\mathbf{A}$ to point $\mathbf{B}$ favours the weak user, since its SE increases by approximately $11 \mathrm{bits} / \mathrm{s} / \mathrm{Hz}$, while the SE of the strong user is reduced by the same amount. However, it represents only a little sacrifice in terms of EE, i.e. 929 bits/J, which underlines that near-optimal $\mathrm{EE}$ and fairness can be achieved at the same.

For a desired $E_{C}$, its corresponding EE contour is obtained by using (24). The intersection of the line of fairness and this contour (i.e. contour labeled 1) will give the optimal SE point. Two intersections can be seen in Figure 1, points $\mathbf{C}$ and $\mathbf{D}$, with both meeting the required criteria in terms of fairness and EE. However, point $\mathbf{D}$ is the desired operating point since both users achieve higher SEs.
TABLE I

SYSTEM PARAMETERS [15], [10], [23]

\begin{tabular}{|c|c|}
\hline Thermal noise $\left(N_{0}\right)$ & $-165.2 \mathrm{dBm} / \mathrm{Hz}$ \\
\hline Bandwidth $(B)$ & $10 \mathrm{MHz}$ \\
\hline Carrier Frequency $\left(f_{c}\right)$ & $2.1 \mathrm{GHz}$ \\
\hline Max. Transmit power $\left(P_{\max }\right)$ & $20 \mathrm{~W}$ \\
\hline BS Circuit power $\left(P_{b s}\right)$ & $130 \mathrm{~W}$ \\
\hline UE Circuit power $\left(P_{u e}\right)$ & $0.1 \mathrm{~W}$ \\
\hline Amplifier efficiency coefficient $(\beta)$ & 4.7 \\
\hline \multirow{2}{*}{ Antenna Gain } & $15 \mathrm{dBi}$ \\
\hline & $-1 \mathrm{dBi}$ \\
\hline \multicolumn{2}{|c|}{$P L_{L O S}(d)=97.4+20 \log _{10}\left(f_{c}\right)+24.2 \log _{10}(d)$} \\
\hline \multicolumn{2}{|c|}{$P L_{N L O S}(d)=125.1+20 \log _{10}\left(f_{c}\right)+42.8 \log _{10}(d)$} \\
\hline \multicolumn{2}{|c|}{$\operatorname{Prob}[L O S]=\min \left\{\frac{0.018}{d}, 1\right\} \times\left(1-e^{-\frac{d}{0.063}}\right)+e^{-\frac{d}{0.063}}$} \\
\hline
\end{tabular}

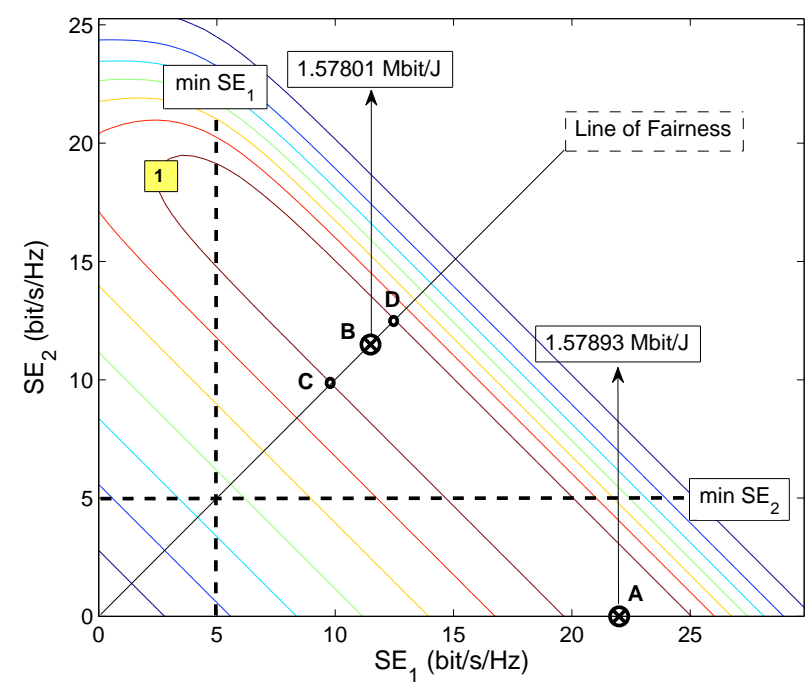

Fig. 1. EE contours with multiple optimality measures.

In order to achieve a minimum user satisfaction in terms of SE, a minimum SE constraint is added to the system. Let us assume that both users in Figure 1 have a minimum SE target of $5 \mathrm{bits} / \mathrm{s} / \mathrm{Hz}$. The dashed lines represent the minimum SE of each user, where the achievable operating point will be the area above the intersection of these two lines. It is very clear that the system can no longer achieve its maximum EE, i.e. point $\mathbf{A}$ will no longer be achievable. On the other hand, the fairness of the system increases, since the user with poor channel quality is able to receive data. The lower the minimum $\mathrm{SE}$ constraint is, the closer the system can operate from the optimal EE value.

\section{Numerical Results AND Discussions}

In this section, we compare the EE, SE, power consumption and fairness of the four EE based resource allocation problems described in Sections III-A and III-B, by considering the sophisticated power model of [15] and a realistic propagation model. Concerning the power model, we assume that $P_{u e}$ is in the order of the circuit power as defined in [10], i.e. $100 \mathrm{~mW}$, and use the values in [15] for the parameters $P_{b s}, P_{\max }, \beta$. A distance-dependent path loss model is employed based on the dense urban macro cell propagation parameters given in [23]. 

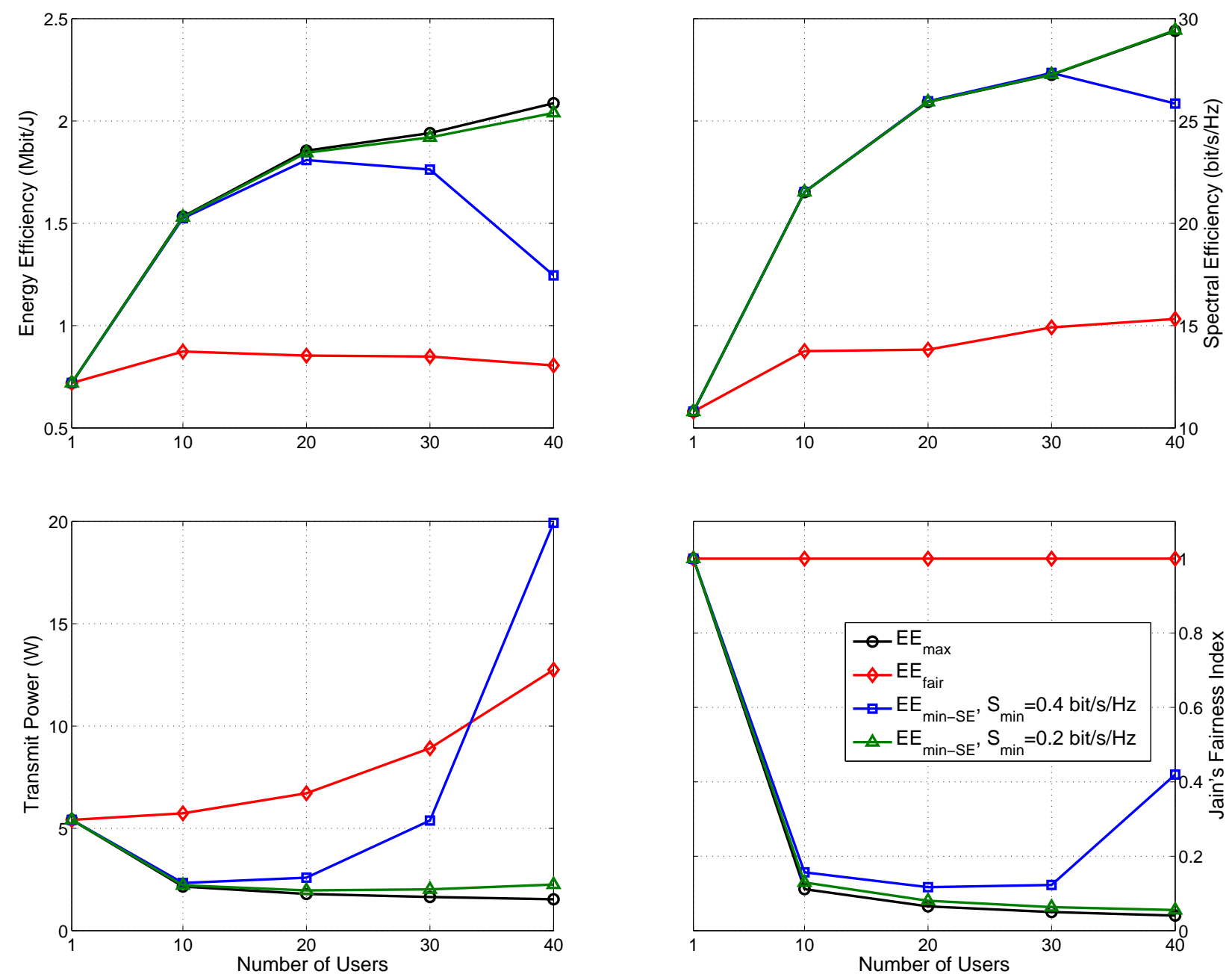

Fig. 2. Performance comparison of multiple resource allocation schemes for different metrics vs. number of users, when $d=1 \mathrm{~km}$.

Both line of sight (LOS) and non-line of sight (NLOS) are considered, where, $P L$ is the path loss in $\mathrm{dB}$ and $d$ is the distance between the transmitter and receiver in kilometres. Our simulation results have been obtained through Monte-Carlo simulations where the users are uniformly distributed within the circular cell. The power and system model parameters are summarized in Table I for the readers convenience.

Figure 2 compares the performance of the three resource allocation methods identified earlier as EE maximization $\left(\mathrm{EE}_{\max }\right)$, EE maximization with rate-fairness $\left(\mathrm{EE}_{\text {fair }}\right)$, and EE maximization with minimum SE constraint $\left(\mathrm{EE}_{\text {min-SE }}\right)$, in terms of different metrics against the number of users for the case where $d=1 \mathrm{~km}$. For the $\mathrm{EE}_{\min -\mathrm{SE}}$ case, two different minimum SE constraints per user have been chosen and the corresponding results are compared.

Results show that increasing the number of users will increase the EE and SE, while the energy consumption decreases, since as $K$ increases, the number of users close to the $\mathrm{BS}$ also increase. The $\mathrm{EE}_{\max }$ scheme achieves the highest EE which is obtained by significantly reducing the total transmit power compared to the other schemes, $\mathrm{EE}_{\text {fair }}$ in particular. In the $\mathrm{EE}_{\min -\mathrm{SE}}$ case however, for low $S_{\text {min }}$ values, the performance is close to the optimal $\mathrm{EE}_{\max }$ scheme, but as $S_{\text {min }}$ increases, the EE significantly reduces for high number of users. It can be seen that a similar trend is also applicable for the $\mathrm{SE}$ of the system where once again the results accentuate the fact that EE maximization of the $\mathrm{BC}$ is extremely spectrally-unfair, which is why the $\mathrm{EE}_{\text {fair }}$ scheme has the worst performance. Moreover, by increasing the number of users, the resource allocation schemes that do not enforce a hard fairness constraint see a sharp reduction in the Jain's fairness index, since the primary target of the system is to maximize EE, therefore favouring new users closer to the BS. Additionally, increasing $S_{\min }$ for the $\mathrm{EE}_{\min -\mathrm{SE}}$ scheme will improve the level of fairness, and full fairness can be achieved by using the $\mathrm{EE}_{\text {fair }}$ scheme, which comes at the cost of lower overall EE and SE.

Figure 3 considers the same resource allocation schemes as before, and compares multiple metrics against the cell radius for the case where $K=40$. It can be seen that by increasing the cell radius, the transmit power required by the BS to convey the information to the UEs increases, and as a result, 

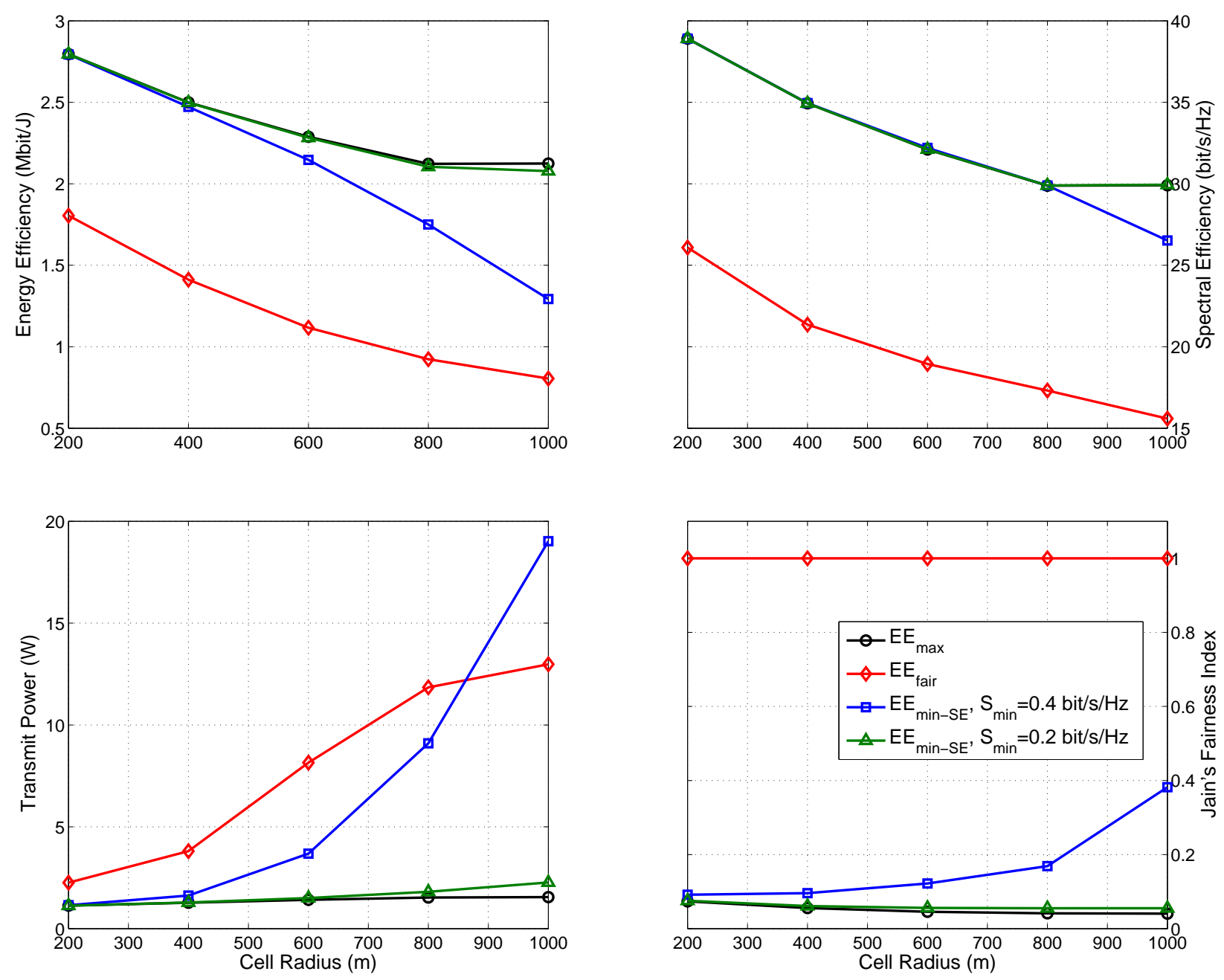

Fig. 3. Performance comparison of multiple resource allocation schemes for different metrics vs. cell radius, when $\mathrm{K}=40$ users.

the EE of the system decreases. Once again, results show that the $\mathrm{EE}_{\max }$ scheme consumes the least amount of energy while having the highest $\mathrm{SE}$ and $\mathrm{EE}$, with $\mathrm{EE}_{\min -\mathrm{SE}}$ (for $S_{\text {min }}=0.2$ $\mathrm{bit} / \mathrm{s} / \mathrm{Hz})$ in close proximity. Moreover, increasing the cell radius will be disadvantageous to users further away from the BS, which will have lower SEs, and therefore unable to receive data. Therefore, the $\mathrm{EE}_{\max }$ scheme achieves a lower level of fairness compared to $\mathrm{EE}_{\text {fair }}$ and $\mathrm{EE}_{\min -\mathrm{SE}}$ (for $S_{\min }=0.4$ $\mathrm{bit} / \mathrm{s} / \mathrm{Hz})$.

\section{CONCLUSION}

This paper presented an optimal energy-efficient resource allocation scheme for the $\mathrm{BC}$, where a sophisticated linear power model was used to define the total consumed power, encompassing the BS transmit power as well as the circuit and processing powers of the BS and UEs. The EE objective function was formulated as a function of the SE, where the proposed resource allocation scheme was further extended to incorporate fairness through Jains fairness measure, and also provide a minimum user satisfaction in terms of SE. We derived a generic equation for the EE contours, which were used to obtain insights into the EE-SE trade-off, where different optimality measures where investigated. Results showed that the network EE increases with the number of users and decrease as the cell radius increases. Moreover, the $\mathrm{EE}_{\max }$ resource allocation scheme showed consistent improvement in terms of $\mathrm{EE}, \mathrm{SE}$ and total transmit power compared to the other schemes.

\section{APPENDIX A}

PRoOF OF CONCAVITY OF $h(\mathbf{X})$

Proof of concavity of $h(\boldsymbol{X})$ for $K=2$ : The Hessian of $h(\mathbf{X})$ can be expressed as:

$$
H(h(\mathbf{X}))=\left[\begin{array}{cc}
\frac{\partial^{2} h(\mathbf{X})}{\partial X_{1}^{2}} & \frac{\partial^{2} h(\mathbf{X})}{\partial X_{1} \partial X_{2}} \\
\frac{\partial^{2} h(\mathbf{X})}{\partial X_{2} \partial X_{1}} & \frac{\partial^{2} h(\mathbf{X})}{\partial X_{2}^{2}}
\end{array}\right]
$$

which further simplifies as:

$$
\begin{aligned}
& H(h(\mathbf{X}))=\frac{1}{f(\mathbf{X})^{2}} \\
& {\left[\begin{array}{ll}
\frac{\partial f(\mathbf{X})}{\partial X_{1}}\left(f(\mathbf{X})-\frac{\partial f(\mathbf{X})}{\partial X_{1}}\right) & \frac{\partial f(\mathbf{X})}{\partial X_{2}}\left(f(\mathbf{X})-\frac{\partial f(\mathbf{X})}{\partial X_{1}}\right) \\
\frac{\partial f(\mathbf{X})}{\partial X_{2}}\left(f(\mathbf{X})-\frac{\partial f(\mathbf{X})}{\partial X_{1}}\right) & \frac{\partial f(\mathbf{X})}{\partial X_{2}}\left(f(\mathbf{X})-\frac{\partial f(\mathbf{X})}{\partial X_{2}}\right)
\end{array}\right]}
\end{aligned}
$$


where,

$$
\left\{\begin{array}{l}
\frac{\partial f(\mathbf{X})}{\partial X_{1}}=f(\mathbf{X})-\alpha_{0} \\
\frac{\partial f(\mathbf{X})}{\partial X_{2}}=f(\mathbf{X})-\alpha_{0}-\alpha_{1} e^{X_{1}}
\end{array}\right.
$$

Let $\mathbf{z}=\left[z_{1}, z_{2}, \ldots, z_{K}\right], \mathbf{z} \in \mathbb{R}^{K}$. It was shown in [21] that if $\mathbf{z} H(h(\mathbf{X})) \mathbf{z}^{T} \leq 0$, then $H(h(\mathbf{X}))$ is a negative semidefinite matrix and consequently according to the second-order condition, $h(\mathbf{X})$ is a concave function. Here,

$$
\begin{array}{rl}
f\left(\mathbf{X}^{2}\right) \mathbf{z} & H(h(\mathbf{X})) \mathbf{z}^{T} \\
=\quad & \left(z_{1}^{2} \alpha_{0}\left(\alpha_{1} e^{X_{1}}+\alpha_{2} e^{X_{1}+X_{2}}\right)\right. \\
& \left.+z_{2}^{2}\left(\alpha_{0}+\alpha_{1} e^{X_{1}}\right) \alpha_{2} e^{X_{1}+X_{2}}+2 z_{1} z_{2} \alpha_{0} \alpha_{2} e^{X_{1}+X_{2}}\right) \\
= & -\left(z_{1}^{2} \alpha_{0} \alpha_{1} e^{X_{1}}+z_{2}^{2} \alpha_{1} \alpha_{2} e^{2 X_{1}+X_{2}}\right. \\
& \left.+\left(z_{1}+z_{2}\right)^{2} \alpha_{0} \alpha_{2} e^{X_{1}+X_{2}}\right)
\end{array}
$$

Thus, $\mathbf{z} H(h(\mathbf{X})) \mathbf{z}^{T} \leq 0, \forall \mathbf{z} \in \mathbb{R}^{K}$ if $\alpha_{0} \alpha_{1}, \alpha_{1} \alpha_{2}$ and $\alpha_{0} \alpha_{2} \geq$ 0 . By definition, $\alpha_{k} \geq 0, \forall k \in[1, \ldots, K]$, so if $\alpha_{0} \geq 0$, the previous condition will be satisfied and $h(\mathbf{X})$ will be concave.

Proof of concavity of $h(\boldsymbol{X})$ for the general $K$-user case: For the general case, it can be easily proven that the $i^{t h}$ row element of the $j^{t h}$ column of the Hessian of $h(\mathbf{X})$, i.e. $H(h(\mathbf{X}))_{i, j}$, can be expressed as:

$H(h(\mathbf{X}))_{i, j}=-\frac{1}{f(\mathbf{X})^{2}}\left[\frac{\partial f(\mathbf{X})}{\partial X_{\max (i, j)}}\left(f(\mathbf{X})-\frac{\partial f(\mathbf{X})}{\partial X_{\min (i, j)}}\right)\right]$

where,

$$
\frac{\partial f(\mathbf{X})}{\partial X_{u}}=f(\mathbf{X})-\left(\alpha_{0}+\sum_{i=1}^{u-1} \alpha_{i} \prod_{j=1}^{i} e^{X_{j}}\right) .
$$

Consequently for the general case, the matrix product $\mathbf{z} H(h(\mathbf{X})) \mathbf{z}^{T}$ can be expressed as:

$$
\begin{aligned}
\mathbf{z} H(h(\mathbf{X})) \mathbf{z}^{T}=-\frac{1}{f(\mathbf{X})^{2}} \sum_{i=0}^{K-1} \sum_{j=1}^{K-i}\left(\sum_{k=0}^{i} z_{j+k}\right)^{2} \\
\alpha_{j-1} \alpha_{j+1} \prod_{m=1}^{j-1} e^{X_{m}} \prod_{n=1}^{j+1} e^{X_{n}}
\end{aligned}
$$

Thus, $\mathbf{z} H(h(\mathbf{X})) \mathbf{z}^{T} \leq 0, \forall \mathbf{z} \in \mathbb{R}^{K}$ if all the possible combinations of $\alpha_{i} \alpha_{j}, \forall i \in[1, \ldots, K-1], j \in[1, \ldots, K]$ and $j>i$ are positive. A necessary and sufficient condition for this to happen is $\alpha_{0} \geq 0$, since by definition, $\alpha_{k} \geq 0, \forall k \in$ $[1, \ldots, K]$.

Consequently, $h(\mathbf{X})$ will be concave if $\alpha_{0} \geq 0$. On the other hand, since $g(\mathbf{X})>0$, and is a linear function, $\ln (g(\mathbf{X}))$ is concave. The sum of two concave functions is concave, therefore we can conclude that $\ln \left(E E_{T}\right)$ is concave, and by implication $E E_{T}$ is log-concave.

\section{APPENDIX B}

PROOF OF OPTIMAL SE POINTS

Proof: Let $E E_{T}$ in (5) be re-expressed as:

$$
E E_{T}(\mathbf{X})=\frac{B}{\ln (2)} \frac{\mathbf{X} \mathbf{1}^{T}}{\beta P(\mathbf{X})+P_{\mathrm{c}}} .
$$

Since $E E_{T}$ is a concave function of $\mathbf{X}$ (see the proof in Appendix A), there exists a unique $\mathbf{X}$ for which $E E_{T}$ is maximized over its entire domain. Let $\mathbf{X}^{\star}$ be this optimum value of $\mathbf{X}$, then we know by definition that $\nabla E E_{T}\left(\mathbf{X}=\mathbf{X}^{\star}\right)=\mathbf{0}$, or equivalently, $\frac{\partial E E_{T}\left(\mathbf{X}=\mathbf{X}^{\star}\right)}{\partial X_{k}}=0$, which in turns implies that:

$$
\beta P\left(\mathbf{X}^{\star}\right)+P_{\mathrm{c}}=\beta \mathbf{X}^{\star} \mathbf{1}^{T} \frac{\partial P\left(\boldsymbol{X}^{\star}\right)}{\partial X_{k}}
$$

since

$$
\begin{aligned}
\frac{\partial E E_{T}(\mathbf{X})}{\partial X_{k}} & =\frac{B}{\ln (2)\left(P(\mathbf{X})+P_{\mathrm{c}}\right)^{2}} \times \\
& \left(\beta P(\mathbf{X})+P_{\mathrm{c}}-\beta \mathbf{X} 1^{T} \frac{\partial P(\boldsymbol{X})}{\partial X_{k}}\right)=0
\end{aligned}
$$

where 1 is a $1 \times K$ vector of ones and $\{.\}^{T}$ is the transpose operator. Identifying the right side of (32) with the left side of (33), the latter can be re-expressed as:

$$
\frac{1}{E E_{T}^{\star}}=\frac{1}{E E_{T}\left(\mathbf{X}^{\star}\right)}=\frac{\beta \ln (2)}{B} \frac{\partial P\left(\boldsymbol{X}^{\star}\right)}{\partial X_{k}}
$$

which in turns implies that

$$
\frac{\partial P\left(\boldsymbol{X}^{\star}\right)}{\partial X_{k}}=\frac{\partial P\left(\boldsymbol{X}^{\star}\right)}{\partial X_{l}}
$$

for any $l \neq k \in\{1, \ldots, K\}$, since $\frac{1}{E E_{T}^{\star}}$ is a constant. Knowing from (4) that

$$
\frac{\partial P(\boldsymbol{X})}{\partial X_{k}}=N \sum_{i=k}^{K} \alpha_{i} \prod_{j=1}^{i} e^{X_{j}}
$$

it implies with (36) that

$$
N \sum_{i=k}^{l-1} \alpha_{i} \prod_{j=1}^{i} e^{X_{j}^{\star}}=0
$$

if $l>k$ and, hence, $X_{j}^{\star} \rightarrow-\infty$ for any $j \in\{k, \ldots, l-1\}$. However, we know that $X_{j} \geq 0$ for any $j \in\{1, \ldots, K\}$; thus, $X_{j}^{\star}=0 j \in\{k, \ldots, l-1\}$. Let $l=K$ and $k=1$, then $X_{j}^{\star}=0 j \in\{1, \ldots, K-1\}$. Inserting the latter result in (33), we obtain that:

$$
\beta N\left[-g_{K}^{-1}+\sum_{i=1}^{K-1} \alpha_{i}+\alpha_{K} e^{X_{K}}\right]+P_{\mathrm{c}}=N \beta X_{K} \alpha_{K} e^{X_{K}}
$$

or equivalently,

$$
e^{-1} \sum_{i=0}^{K-1} \alpha_{i}=\left(X_{K}-1\right) \alpha_{K} e^{X_{K}-1}
$$

since $\alpha_{0}=P_{\mathrm{c}} /(N \beta)-g_{K}^{-1}$. Consequently, the $K^{\text {th }}$ user optimal SE is given by

$$
X_{K}^{\star}=\mathcal{W}_{0}\left(e^{-1}\left(\frac{g_{1} P_{\mathrm{c}}}{N \beta}-1\right)\right)+1
$$

since $\sum_{i=0}^{K-1} \alpha_{i}=P_{\mathrm{c}} /(N \beta)-g_{1}^{-1}$ and $\alpha_{K}=g_{1}^{-1}$. 


\section{APPENDIX C}

\section{EE CONTOUR DERIVATION}

Proof: Using $E_{C}$ and (7), the total EE function can be expressed as:

$$
\begin{aligned}
A^{-1} E_{C}\left(\alpha_{0}\right. & \left.+\sum_{k=1}^{K-1} \alpha_{k} \prod_{j=1}^{k} e^{X_{j}}\right) \\
& =\sum_{k=1}^{K} X_{k}-A^{-1} E_{C} \alpha_{K} \prod_{k=1}^{K} e^{X_{k}} .
\end{aligned}
$$

Therefore, we obtain that

$$
\begin{aligned}
& -A^{-1} E_{C} \alpha_{K} e^{A^{-1} E_{C}\left(\alpha_{0}+\sum_{k=1}^{K-1} \alpha_{k} \prod_{j=1}^{k} e^{X_{j}}\right)} \\
& \quad=-A^{-1} E_{C} \alpha_{K} \prod_{k=1}^{K} e^{X_{k}} e^{-A^{-1} E_{C} \alpha_{K} \prod_{k=1}^{K} e^{X_{k}} .}
\end{aligned}
$$

By using the Lambert function, it implies that

$$
\begin{aligned}
& -A^{-1} E_{C} \alpha_{K} \prod_{k=1}^{K} e^{X_{k}} \\
& =\mathcal{W}\left[-A^{-1} E_{C} \alpha_{K} e^{A^{-1} E_{C}\left(\alpha_{0}+\sum_{k=1}^{K-1} \alpha_{k} \prod_{j=1}^{k} e^{X_{j}}\right)}\right]
\end{aligned}
$$

By rearranging the latter, it can finally be re-expressed

$$
\begin{aligned}
& X_{K}= \\
& \ln \left\{\frac{-\mathcal{W}\left[-A^{-1} E_{C} \alpha_{K} e^{\left.A^{-1} E_{C}\left(\alpha_{0}+\sum_{k=1}^{K-1} \alpha_{k} \prod_{j=1}^{k} e^{X_{j}}\right)\right]}\right.}{A^{-1} E_{C} \alpha_{K} \prod_{k=1}^{K-1} e^{X_{k}}}\right\}
\end{aligned}
$$

\section{REFERENCES}

[1] M. Gruber, O. Blume, D. Ferling, D. Zeller, M. A. Imran, E. C. Strinati, "EARTH-Energy Aware Radio and Network Technologies," in proc. IEEE Personal, Indoor and Mobile Radio Communications, PIMRC'09, Tokyo, Japan, pp. 1-5, Sept. 2009.

[2] F. Richter, A. J. Fehske, G. P. Fettweis, "Energy Efficiency Aspects of Base Station Deployment Strategies for Cellular Networks," in proc. IEEE Vehicular Technology Conference Fall, VTC'09-Fall, Anchorage, USA, pp. 1-5, Sept. 2009.

[3] S. Vadgama,"Trends in green wireless access," Fujitsu Scientific \& Tech. Journal, vol. 45, no. 4, pp. 404-408, Oct. 2009.

[4] W. Rhee, J. Cioffi, "Increase in capacity of multiuser OFDM system using dynamic subchannel allocation," in proc. IEEE Vehicular Technology Conference, VTC'00, Tokyo, Japan, pp. 1085-1089, May 2000.

[5] C. Wong, R. Cheng, K. Lataief, R. Murch, " Multiuser OFDM with adaptive subcarrier, bit, and power allocation," IEEE Journal on Selected Areas in Communications, vol. 17, no.10, pp. 1747-1758, Oct. 1999.

[6] T. Keller and L. Hanzo, "Adaptive multicarrier modulation: a convenient framework for time frequency processing in wireless communications," in proc. of the IEEE, vol. 88, no. 5, pp. 611-640, May 2000.

[7] M. J. E. Golay, "Note on the theoretical efficiency of information reception with PPM," IRE 1949, vol. 37, no. 1, pp.1031, Sept. 1949.

[8] H. M. Kwon, T. G. Birdsall, "Channel Capacity in Bits per Joule," IEEE Journal of Oceanic Engineering, vol. 0E-11, no. 1, pp.97-99, Jan. 1986.

[9] G. W. Miao, N. Himayat, Y. Li, D. Bormann, "Energy-efficient design in wireless OFDMA," in proc. IEEE International Conference on Communications, ICC'08, Beijing, China, pp.3307-3312, May 2008.

[10] G. W. Miao, N. Himayat, Y. Li, "Energy-Efficient Link Adaptation in Frequency-Selective Channels,"IEEE Transaction on Wireless Coтmunications, vol. 58, no. 2, pp.545-554, Feb. 2010.

[11] F. Héliot, M. A. Imran, and R. Tafazolli, "Energy-efficiency based resource allocation for the orthogonal multi-user channel," in Proc. IEEE Vehicular Technology Conference Fall, VTC'12-Fall (to appear), Québec city, Canada, Sep. 2012.
[12] J. L. Gao, "Analysis of Energy Consumption of Ad Hoc Wireless Sensor Networks Using a Bit-Meter-per-Joule Metric," IPN Progress Report, pp.42-150, Aug. 2002.

[13] A. Akbari, M. A. Imran, R. Hoshyar, A. Amich, and R. Tafazolli, "Average Energy Efficiency Contours with Multiple Decoding Policies," IEEE Communications Letter, vol. 15, no. 5, pp. 506-508, May 2011.

[14] R. Jain, D. M. Chiu, and W. Hawe, " A Quantitative Measure of Fairness and Discrimination for Resource Allocation in Shared Systems," DEC Research Report TR-301, 1984.

[15] G. Auer, et al., "How Much Energy is Needed to Run a Wireless Netwrok?," IEEE Wireless Communications Magazine, vol. 18, no. 5, pp.40-49, Oct. 2011.

[16] A. J. Goldsmith, "The capacity of downlink fading channels with variable rate and power," IEEE Transactions on Vehicular Technology, vol. 46, no.3, pp. 1747-1758, Aug. 1997.

[17] A. Goldsmith, Wireless Communications. Cambridge University press, 2005.

[18] P. P. Bergmans, "A simple converse for broadcast channels with additive white Gaussian noise," IEEE Transaction on Information Theory, vol. 20, no. 2, pp.279-280, March 1974.

[19] A. J. Fehske, G. P. Fettweis, J. Malmodin, and G. Biczok "The global footprint of mobile communications: The ecological and economic perspective," IEEE Communications Magazine, vol. 49, no. 8, pp.55-62, Aug. 2011.

[20] G. Auer, et al., "D2.3:Energy Efficiency Analysis of the Reference Systems, Areas of Improvements and Target Breakdown," INFSO-ICT247733 EARTH (Energy Aware Radio and NeTwork TecHnologies), Tech. Rep., Nov. 2010.

[21] S. Boyd and L. Vandenberghe, Convex Optimization. Cambridge University Press, 2004.

[22] R. Courant and H. Robbins, What is Mathematics?: An Elementary Approach to Ideas and Methods. New York: Oxford University Press, 1996.

[23] A. J. Fehske, P. Marsch, and G. P. Fettweis, "Bit per joule efficiency of cooperating base stations in cellular networks," in proc. IEEE Global Communications Conference, GLOBECOM'10, 3rd International Workshop on Green Communications, Florida, Dec. 2010.

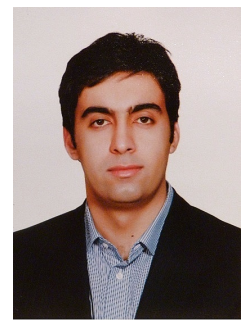

Amir Akbari (S'09) received the B.Eng. degree in telecommunication engineering with first class honours from King's College London, UK, in 2006, the M.Sc. degree in digital signal processing from King's College London, UK, in 2007, and the Ph.D. degree in mobile communications from the University of Surrey, UK, in 2012. He is currently a research fellow at the Centre for Communication Systems Research (CCSR), University of Surrey. He has been involved in European Commission funded research project EARTH, and is currently involved in the GreenComm project with Huawei. His main research interests include energy efficiency of wireless networks and radio resource allocation in multiuser communication networks.

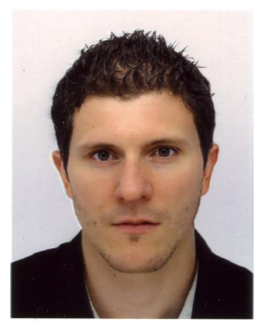

Fabien Héliot (S'05-M'07) received the M.Sc degree in Telecommunications from the Institut Supérieur de l'Electronique et du Numérique (ISEN), Toulon, France, and the Ph.D. degree in Mobile Telecommunications from King's College London, in 2002 and 2006, respectively. He is currently a research fellow at the Centre for Communication Systems Research (CCSR) of the University of Surrey. He has been actively involved in European Commission funded projects such as IST FIREWORKS and IST ROCKET projects. He is currently involved in the award-winning ICT EARTH project, which is a leading European project investigating the energy efficiency of mobile communication systems. His main research interests are energy efficiency, cooperative communication, MIMO, and radio resource management. 


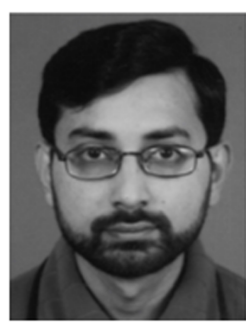

Muhammad Ali Imran (SM'12) received his B.Sc. degree in Electrical Engineering from University of Engineering and Technology Lahore, Pakistan, in 1999, and the M.Sc. and Ph.D. degrees from Imperial College London, UK, in 2002 and 2007, respectively. He secured first rank in his BSc and a distinction in his MSc degree along with an award of excellence in recognition of his academic achievements, conferred by the President of Pakistan. He is currently a lecturer in the Centre for Communication Systems Research (CCSR) at the University of Surrey, UK. He has been actively involved in European Commission funded research projects ROCKET and EARTH, Mobile VCE funded project on fundamental capacity limits and EPSRC funded project India UK ATC. He is the principal investigator of EPSRC funded REDUCE project. His main research interests include the analysis and modeling of the physical layer, optimization for the energy efficient wireless communication networks and the evaluation of the fundamental capacity limits of wireless networks.

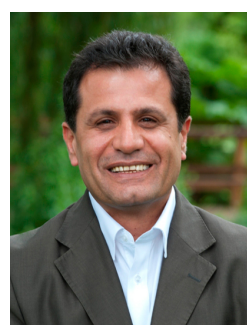

Rahim Tafazolli (SM'09) is a professor and the Director of Centre for Communication Systems Research (CCSR), University of Surrey, United Kingdom. He has been active in research for more than 20 years and has authored and co-authored more than 360 papers in refereed international journals and conferences. Professor Tafazolli is a consultant to many mobile companies, has lectured, chaired, and been invited as keynote speaker to a number of IET and IEEE workshops and conferences. He has been Technical Advisor to many mobile companies, all in the field of mobile communications. He is the Founder and past Chairman of IET International Conference on 3rd Generation Mobile Communications. $\mathrm{He}$ is a Fellow of the IET and WWRF (Wireless World Research Forum). He is Chairman of EU Expert Group on Mobile Platform (e-mobility SRA) and Chairman of Post-IP working group in e-mobility, past Chairman of WG3 of WWRF. He is nationally and internationally known in the field of mobile communications. 